\title{
ON THE EXISTENCE OF INVARIANT SUBSPACES IN SPACES WITH INDEFINITE METRIC ${ }^{1}$
}

\author{
KYUYA MASUDA
}

\begin{abstract}
Let $\boldsymbol{P}_{1}, \boldsymbol{P}_{\mathbf{2}}$ be complementary projections in Hilbert space $\boldsymbol{H}$. Let $U$ be a one-to-one and onto operator in $\boldsymbol{H}$ with $Q(U x)=Q(x)$, where $Q(x)=\left\|P_{1} x\right\|^{2}-\left\|P_{2} x\right\|^{2}$. The sufficient condition is given for the unique existence of maximal subspace $\boldsymbol{L}$ invariant under all operators commuting with $U$, and such that $Q(x) \geqq 0, x \in \boldsymbol{L}$. The result was obtained in the course of attacking the problem proposed by Phillips [5] (see also [1]).
\end{abstract}

Let $\boldsymbol{H}$ be a (separable) complex Hilbert space, with the ordinary scalar product $(\cdot, \cdot)$, and with indefinite metric $Q(x, y)=(J x, y)\left(J=P_{1}-P_{2}\right.$; $P_{1}, P_{2}$ are two complementary orthogonal projections). Let $\mathscr{K}$ be the set of all bounded linear operators $K$ from $H$ to $P_{2} H\left(\equiv H_{2}\right)$ with a bound $\leqq 1$ and such that $K \boldsymbol{H}_{2}=0$. Let $\mathscr{M}$ be the set of all maximal positive subspaces $\boldsymbol{M}$ in $\boldsymbol{H} ; M$ is said to be positive if $Q(x, x) \geqq 0$ for $x$ in $\boldsymbol{M}$. Let $\mathscr{C}(T)$ be the set of all bounded linear operators that commute with $T$ and its $Q$ adjoint operator $T^{\circ} ; T^{\circ}$ is defined by $Q\left(T^{\circ} x, y\right)=Q(x, T y) . U$ is called a $Q$ unitary operator if $Q(U x, U y)=Q(x, y)$ and if $U$ is onto and one-to-one. Let $\boldsymbol{H}_{j}=\boldsymbol{P}_{j} \boldsymbol{H}(j=1,2)$. Let $U_{j k}=P_{j} U P_{k}(j, k=1,2)$. Now our main result is the following:

THEOREM. Let $U$ be a $Q$-unitary operator. Suppose that there exist convex open sets $\Omega_{1}, \Omega_{2}$ with $\bar{\Omega}_{1} \cap \bar{\Omega}_{2}=\varnothing(\bar{\Omega}$ means the closure of $\Omega)$ such that

$$
\begin{aligned}
& \left\{\left(U(1+K) x_{1}, x_{1}\right) ;\left\|x_{1}\right\|=1, x_{1} \in \boldsymbol{H}_{1}\right\} \subset \Omega_{1}, \\
& \left\{\left((1-K) U x_{2}, x_{2}\right) ;\left\|x_{2}\right\|=1, x_{2} \in \boldsymbol{H}_{2}\right\} \subset \Omega_{2},
\end{aligned}
$$

for all $K$ in $\mathscr{K}$. Then there exists a unique maximal positive subspace invariant under $T$ in $\mathscr{C}(U)$.

Corollary. Suppose that $A$ is a bounded $Q$-selfadjoint operator $\left(A=A^{\circ}\right)$ satisfying the condition $(*)$, given in the above theorem, with $U$ replaced by $A$. Then there exists a unique maximal positive subspace invariant under $T$ in $\mathscr{C}(A)$.

Received by the editors July 30, 1970 and, in revised form, June 28, 1971.

AMS 1969 subject classifications. Primary 4735; Secondary 4615.

Key words and phrases. Maximal positive invariant subspace, indefinite metric space.

${ }^{1}$ This research was done while the author was at Stanford University, and was supported by NSF Grant SP 16115.

(c) American Mathematical Society 1972 
REMARK. The following example shows that there exist, generally, more than one invariant subspace in $\mathscr{M}$ when the condition $\left(^{*}\right)$ is violated. Let $\boldsymbol{H}$ be a two-dimensional complex euclidean space with element $x=\left\{x_{1}, x_{2}\right\}$. Define projections $P_{1} x=\left\{x_{1}, 0\right\}, P_{2} x=\left\{0, x_{2}\right\}$, and let $A$ be an operator defined by $A x=\left\{\lambda x_{1}+x_{2},-x_{1}\right\}$ ( $\lambda$ :real). Then the $A_{\lambda}$ satisfies all the conditions in the corollary if $|\lambda|>2$. Hence for $|\lambda|>2$ there exists a unique maximal positive subspace $\boldsymbol{M}_{\lambda}$ invariant under $\mathscr{C}\left(A_{\lambda}\right)$. On the other hand, by direct calculation, we can see that the set $\mathscr{M}_{\lambda}$ of all maximal positive subspaces invariant under $\mathscr{C}\left(A_{\lambda}\right)$ consists of $[\{x,(-\lambda+$ $\left.\left.\left.\left(\lambda^{2}-4\right)^{1 / 2}\right) x / 2\right\} ; x \in C\right]$ for $\lambda>2 ;\left[\left\{x,\left(-\lambda \pm\left(\lambda^{2}-4\right)^{1 / 2}\right) x / 2\right\} ; x \in C\right]$ for $|\lambda| \leqq 2$; $\left[\left\{x,\left(-\lambda-\left(\lambda^{2}-4\right)^{1 / 2}\right) x / 2\right\} ; x \in C\right]$ for $\lambda<-2$.

Proof of THEOREM. By the $Q$-unitarity of $U$,

Hence,

$$
\begin{aligned}
\|x\|^{2}+\left\|\left(U_{21}+z U_{22} K\right) x\right\|^{2} & \leqq|z|^{2}\|K x\|^{2}+\left\|\left(U_{11}+z U_{12} K\right) x\right\|^{2} \\
& \leqq|z|^{2}\|x\|^{2}+\left\|\left(U_{11}+z U_{12} K\right) x\right\|^{2} .
\end{aligned}
$$

$$
\left(1-|z|^{2}\right)\|x\|^{2}+\left\|\left(U_{21}+z U_{22} K\right) x\right\|^{2} \leqq\left\|\left(U_{11}+z U_{12} K\right) x\right\|^{2}
$$

for $x$ in $\boldsymbol{H}_{1}, K$ in $\mathscr{K}$ and $|z| \leqq 1$. Using (1), we shall show that for each $K$ in $\mathscr{K}$ and $|z|<1,\left(U_{11}+z U_{12} K\right)^{-1}$ exists as a bounded operator on $\boldsymbol{H}_{1}$ with a bound

$$
\left\|\left(U_{11}+z U_{12} K\right)^{-1}\right\| \leqq\left(1-|z|^{2}\right)^{-1 / 2} .
$$

Indeed, $U_{11}+z U_{12} K$ is, by (1), one-to-one.

If $y$ is orthogonal to $R\left(U_{11}+z U_{12} K\right)(\boldsymbol{R}(T)=$ the range of $T)$, then

$$
\begin{aligned}
0 & =\left(y,\left(U_{11}+z U_{12} K\right) x\right)=Q(y, U(1+z K) x) \\
& =Q\left(U^{\circ} y,(1+z K) x\right)=\left(\left[P_{1} U^{\circ}-\bar{z} K^{*} P_{2} U^{\circ}\right] y, x\right)
\end{aligned}
$$

for all $x$ in $\boldsymbol{H}_{1} ; K^{*}$ is the adjoint operator of $K$ with respect to the ordinary scalar product (note that $K^{*} \boldsymbol{H}_{1}=0, \boldsymbol{R}\left(K^{*}\right) \subseteq \boldsymbol{H}_{1}$, and $\left\|K^{*}\right\| \leqq 1$ ). Hence $\left[P_{1} U^{\circ}-\bar{z} K^{*} P_{2} U^{\circ}\right] y=0$, from which it follows that $\left\|P_{1} U^{\circ} y\right\| \leqq|z|\left\|K^{*}\right\|$ - $\left\|P_{2} U^{\circ} y\right\| \leqq\left\|P_{2} U^{\circ} y\right\|$. Hence $\|y\|^{2}=Q(y, y)=Q\left(U^{\circ} y, U^{\circ} y\right)=\left\|P_{1} U^{\circ} y\right\|^{2}-$ $\left\|P_{2} U^{\circ} y\right\|^{2} \leqq 0$. Here we used the $Q$-unitarity of $U^{\circ}$ implied by that of $U$. Hence $y=0$, i.e., the range of $U_{11}+z U_{12} K$ is dense in $H_{1}$. Thus $U_{11}+z U_{12} K$ is, by (1), onto. The desired bound of $\left(U_{11}+z U_{12} K\right)$ follows from (1).

By Phillips [5], $\boldsymbol{M} \in \mathscr{M}$ if and only if $\boldsymbol{M}=\left\{x_{1}+K x_{1} ; x_{1} \in \boldsymbol{H}_{1}\right\}$ for some $K$ in $\mathscr{K}$. Then it is easy to see that $\boldsymbol{M}$ is invariant under $U$ if and only if $K$ satisfies

$$
K U_{12} K+K U_{11}-U_{22} K-U_{21}=0 .
$$

To find a solution $K$ of (3) in $\mathscr{K}$, we consider instead the equation:

$$
z K(z) U_{12} K(z)+K(z) U_{11}-z U_{22} K(z)-U_{21}=0
$$


in $|z|<1$ and construct $K(z)$ from a sequence of holomorphic functions $\left\{K_{j}(z)\right\}$. We set

$$
K_{0}(z)=0 \text { and } K_{j+1}(z)=\left(U_{21}+z U_{22} K_{j}(z)\right) \cdot\left(U_{11}+z U_{12} K_{j}(z)\right)^{-1}
$$

i.e.,

$$
K_{j+1}(z)\left(U_{11}+z U_{12} K_{j}(z)\right)=U_{21}+z U_{22} K_{j}(z) .
$$

Then the $K_{j}(z)$ has the properties:

(i) $K_{j}(z) \in \mathscr{K}$;

(ii) $K_{j}(z)$ is holomorphic in $|z|<1$;

(iii) for any compact set $\Delta$ in $|z|<1 \sup _{\Delta}\left\|K_{j}^{\prime}(z)\right\|$ is uniformly bounded in $j\left(K^{\prime}:\right.$ derivative in $z$ of $\left.K\right)$;

(iv) $K_{j}(z)$ converges uniformly to some bounded operator $\tilde{K}(z)$ on some small disk $|z|<\varepsilon$ in the operator norm.

Indeed, if $K_{j}(z) \in \mathscr{K}$, then we have, by (1),

$$
\left\|K_{j+1}\left(U_{11}+z U_{12} K_{j}\right) x\right\|^{2}=\left\|\left(U_{21}+z U_{22} K_{j}\right) x\right\|^{2} \leqq\left\|\left(U_{11}+z U_{12} K_{j}\right) x\right\|^{2},
$$

showing (i). Suppose that $K_{j}(z)$ is holomorphic in $|z|<1$. Then from (2) it is easy to see that $\left(U_{11}+z U_{12} K_{j}\right)^{-1}$ is holomorphic in $|z|<1$. Hence $\left(U_{21}+z U_{22} K_{j}(z)\right)\left(U_{11}+z U_{12} K_{j}(z)\right)^{-1}$ is holomorphic in $z$, showing (ii). (iii) is a direct consequence of (ii). Since

$$
\begin{aligned}
K_{j+1}(z)-K_{j}(z)= & z U_{22}\left(K_{j}-K_{j-1}\right)\left(U_{11}+z U_{12} K_{j}\right)^{-1} \\
& +z^{2}\left(U_{21}+z U_{22} K_{j-1}\right)\left(U_{11}+z U_{12} K_{j-1}\right)^{-1} \\
& \cdot U_{12}\left(K_{j}-K_{j-1}\right)\left(U_{11}+z U_{12} K_{j}\right)^{-1},
\end{aligned}
$$

we have, by (2) and (i),

$$
\left\|K_{j+1}-K_{j}\right\| \leqq \frac{1}{2}\left\|K_{j}-K_{j-1}\right\|
$$

for sufficiently small $|z| \leqq \varepsilon$. Hence $K(z)$ converges to some $\tilde{K}(z) \in \mathscr{K}$ uniformly on $|z| \leqq \varepsilon$ in the operator norm. Thus we have (iv).

By (i), (ii), (iii), there exists a subsequence $K_{j^{\prime}}(z)$ converging (in the weak topology) uniformly on any compact set in $|z|<1$ to some holomorphic (in $|z|<1$ ) operator-valued function $K(z) \in \mathscr{K}$. By (iv), $\tilde{K}(z)=K(z)$ in $|z|<\varepsilon$. Since $K_{j}(z)$ converges strongly to $\widetilde{K}(z)$ in $|z|<\varepsilon$ and since $K_{j}$ satisfies (5), $\widetilde{K}(z)$, and so $K(z)$, satisfies (4) in $|z|<\varepsilon$. Since $K(z)$ is weakly, and so strongly, holomorphic in $|z|<1, K(z)$ must satisfy (4) in the whole unit disk $|z|<1$. Now we claim:

(v) $K^{\prime}(z)$ is uniformly bounded for real $z$ in $0<z<1$.

(vi) Equation (3) has at most one solution.

For the moment we assume that (v) and (vi) hold. Then $K(z)$ has a strong limit $K(1) \in \mathscr{K}$ for $z \rightarrow 1(0<z<1)$. Letting $z \rightarrow 1$ in (4), we have

$$
K(1) U_{12} K(1)+K(1) U_{11}-U_{22} K(1)-U_{21}=0 .
$$


Thus the subspace $\boldsymbol{M}=\left\{x_{1}+K(1) x_{1} ; x_{1} \in \boldsymbol{H}_{1}\right\} \in \mathscr{M}$ is invariant under $U$. Besides $M$ is invariant also under $T$ in $\mathscr{C}(U)$. Indeed, if $T$ is in $\mathscr{C}(U)$, then $T+T^{\circ}$ is a $Q$-selfadjoint operator in $\mathscr{C}(U)$; note that $T^{\circ} \in \mathscr{C}(U)$ if $T \in \mathscr{C}(U)$. Hence the operator $V(t)$ defined by $V(t)=\exp \left[i t\left(T+T^{\circ}\right)\right]$ is $Q$-unitary. Hence $V(t)$ maps $M$ onto $M$. Since $U V(t) M=V(t) U M \subset V(t) M$, the space $V(t) M$ in $\mathscr{M}$ is invariant under $U$. By the uniqueness of the solution of (6) (see vi), which implies the uniqueness of invariant maximal positive subspace, we have $V(t) M=M$. Hence $(i t)^{-1}[V(t)-I] M \subset M$. Letting $t \rightarrow 0$, we have $\left(T+T^{\circ}\right) M \subset M$. Similarly $i\left(T-T^{\circ}\right) M \subset M$. Thus $T M \subset M$.

Proof of (v). Since $\bar{\Omega}_{1}, \bar{\Omega}_{2}$ are closed, convex and disjoint sets, there exist $\theta(-\pi / 2 \leqq \theta \leqq \pi / 2), \alpha_{1}, \alpha_{2}\left(\alpha_{1}>\alpha_{2}\right)$ such that

or

$$
\operatorname{Re}\left(e^{i \theta} \zeta_{1}\right)>\alpha_{1} \text { and } \operatorname{Re}\left(e^{i \theta} \zeta_{2}\right)<\alpha_{2}
$$

for $\zeta_{1}$ in $\bar{\Omega}_{1}$ and $\zeta_{2}$ in $\bar{\Omega}_{2}$. We consider only the case (6); the case (7) can be treated similarly. Then by the assumption $(*)$,

$$
\begin{aligned}
& \operatorname{Re}\left(e^{i \theta} P_{1} U(1+K) x_{1}-\alpha_{1} x_{1}, x_{1}\right) \geqq 0, \\
& \operatorname{Re}\left(e^{i \theta} P_{2}(1-K) U x_{2}-\alpha_{2} x_{2}, x_{2}\right) \leqq 0,
\end{aligned}
$$

for all $K \in \mathscr{K}, x_{1} \in H_{1}$ and $x_{2} \in H_{2}$. By this "dissipativity condition",

$$
\begin{aligned}
\left\|\exp \left[-t e^{i \theta} P_{1} U(1+z K)+t \alpha_{1}\right] P_{1}\right\| & \leqq 1 ; \\
\left\|\exp \left[t e^{i \theta} z P_{2}(1-K) U-t z \alpha_{2}\right] P_{2}\right\| & \leqq 1
\end{aligned}
$$

for all $t>0, K \in \mathscr{K}$ and $0<z \leqq 1$. Differentiation in $z$ of (4) gives

i.e.,

$$
K^{\prime}(z)\left(U_{11}+z U_{12} K(z)\right)+z\left(K(z) U_{12}-U_{22}\right) K^{\prime}(z)=B(z)
$$

$$
K^{\prime}(z) P_{1} U(1+z K(z)) P_{1}+z P_{2}(K(z)-1) U P_{2} K^{\prime}(z)=B(z),
$$

where $B(z)=-K(z) U_{12} K(z)+U_{22} K(z)$. Hence

$$
\begin{aligned}
K^{\prime}(z)=-e^{-i \theta} \lim _{N \rightarrow \infty} & \int_{0}^{N} e^{-\left(\alpha_{1}-z \alpha_{2}\right) t} \exp \left[t e^{i \theta} z P_{2}(1-K(z)) U P_{2}-\alpha_{2} z t\right] \\
\cdot & P_{2} \cdot B(z) \cdot \exp \left[-t e^{i \theta} \cdot P_{1} U(1+z K(z)) P_{1}+\alpha_{1} t\right] P_{1} d t
\end{aligned}
$$

Hence, by (8),

$$
\left\|K^{\prime}(z)\right\| \leqq\|B(z)\| \cdot\left(\alpha_{1}-\alpha_{2}\right)^{-1} \leqq\left(\alpha_{1}-\alpha_{2}\right)^{-1}\left(\left\|U_{12}\right\|+\left\|U_{22}\right\|\right)
$$

for $0<z<1$.

Proof of (vi). We consider only the case (6). Let $K_{1}, K_{2}$ be solutions of (3) in $\mathscr{K}$. Then $K_{0}=K_{1}-K_{2}$ satisfies $K_{0}\left(U_{12} K_{2}+U_{11}\right)=\left(U_{22}-K_{1} U_{12}\right) K_{0}$. 
Hence we have

$$
\begin{aligned}
K_{0}= & -e^{-i \theta} \int_{0}^{N} \frac{d}{d t}\left\{\exp \left[t e^{i \theta} P_{2}\left(1-K_{1}\right) U P_{2}\right] P_{2} K_{0}\right. \\
& \left.\cdot \exp \left[-t e^{i \theta} P_{1} U\left(1+K_{2}\right)\right] P_{1}\right\} d t \\
& +e^{-i \theta} e^{-\left(\alpha_{1}-\alpha_{2}\right) N} \exp \left[N e^{i \theta} P_{2}\left(1-K_{1}\right) U P_{2}-\alpha_{2} N\right] \\
& \cdot P_{2} K_{0} \exp \left[-N e^{i \theta} P_{1} U\left(1+K_{2}\right) P_{1}+\alpha_{1} N\right] P_{1} \\
= & e^{-i \theta-\left(\alpha_{1}-\alpha_{2}\right) N} \exp (\cdots) K_{0} \exp (\cdots) .
\end{aligned}
$$

Letting $N \rightarrow \infty$, we have, by (9), $K_{0}=0$. Thus the proof is completed.

Proof of CORollary. The $Q$-selfadjoint operator $A$ defines the oneparameter family of group $U(t)=e^{i t A}$ of $Q$-unitary operators. Then we have

$$
U(t)=I+(i t A)+\frac{1}{2 !}(i t A)^{2}+\cdots .
$$

Hence there exists a $t_{0}$ such that, for each $0<t<t_{0}, U(t)$ satisfies the condition $\left({ }^{*}\right)$. By the theorem, for each $0<t<t_{0}$ there exists the unique subspace $\boldsymbol{M}$ in $\mathscr{M}$ invariant under $\mathscr{C}(U(t))$. Since $\mathscr{C}(A) \subset \mathscr{C}(U(t)), \boldsymbol{M}$ is invariant under $\mathscr{C}(A)$. Conversely if $\boldsymbol{M}(\in \mathscr{M})$ is invariant under $\mathscr{C}(A)$, then it is also invariant under $\mathscr{C}(U(t))$ by the above expansion. Hence the $M$ is the unique subspace in $\mathscr{M}$ invariant under $\mathscr{C}(A)$.

ACKNOWLEDGEMENT. The author expresses his gratitude to the referee for his kind advice, thanks to which the author could remove a superfluous assumption in the theorem.

\section{REFERENCES}

1. J. W. Helton, Unitary operators on a space with an indefinite inner product, J. Functional Analysis 6 (1970), 412-440

2. M. G. Krein, A new application of the fixed-point principle in the theory of operators on a space with indefinite metric, Dokl. Akad. Nauk SSSR 154 (1964), 1023-1026= Soviet Math. Dokl. 5 (1964), 224-228. MR 29 \#6314.

3. H. Langer, Eine Verallgemeinerung eines Satzes von L. S. Pontrjagin, Math. Ann. 152 (1963), 434-436. MR 28 \#1492.

4. M. A. Naĭmark, On commuting unitary operators in spaces with indefinite metric, Acta Sci. Math. (Szeged) 24 (1963), 177-189. MR 28 \#4367.

5. R. S. Phillips, On dissipative operators, Lecture Series in Differential Equations, Van Nostrand, Princeton, N.J., 1969.

Department of Mathematics, Stanford University, Stanford, California 94305

Department of Mathematics, University of Tokyo, Tokyo 113, JaPan (Current address) 